\title{
Influencia de las tecnologías de la información y la comunicación en la gestión interna de las empresas asesoradas por el Centro de Desarrollo de la Micro y Pequeña Empresa de Santa Ana
}

Carlos Edmundo Sayes ${ }^{1}$

Vidal Enrique Cerritos Magaña²

Docentes investigadores, Facultad de Ciencias Empresariales

Universidad Católica de El Salvador, El Salvador

Fecha de recepción: 05-12-2019

Fecha de aceptación: $15-02-2020$

\section{Resumen}

El estudio se centró en el empleo de la tecnología dentro de la gestión interna de las empresas, comprobando cuáles se están utilizando para la promoción y venta de los productos y/o servicios. Se identificó la utilización de la tecnología, así como las limitaciones experimentadas, el plan para la adopción y su seguimiento.

Se describe el nivel de influencia que están teniendo las tecnologías de la información y comunicación en la gestión interna de las empresas asesoradas por el Centro de Desarrollo de Micro y Pequeñas Empresas en la Universidad Católica de El Salvador, sede Santa Ana. Con base en los resultados, se proponen medidas que podrían tomar los empresarios para optimizar el uso de los recursos tecnológicos.

Palabras clave: Empresas, tecnologías, redes sociales, CDMYPE, internet.

\begin{abstract}
The study focused on the use of technology within the internal management of companies, checking which ones are being used for the promotion and sale of products and / or services. The use of technology was identified, as well as the limitations experienced, the plan for adoption and its follow-up.

The level of influence that information and communication technologies are having on the internal management of companies advised by the Micro and Small Business Development Center at Universidad Católica de El Salvador, Santa Ana headquarters is described. With base on the Results, measures are proposed that entrepreneurs could take to optimize the use of technological resources.
\end{abstract}

Key words: Companies, technologies, social networks, CDMYPE, internet.

1. Maestro en Asesoría Educativa; email: carlos.sayes@catolica.edu.sv; ORCID: https://orcid.org/0000-0001-5035-7268

2. Licenciado en Computación Administrativa Empresarial; email: vidal.cerritos@catolica.edu.sv; ORCID: https://orcid. org/0000-0002-0764-5448 


\section{Introducción}

El modelo de Centro de Desarrollo de Micro y Pequeñas Empresas (CDMYPE) $)^{3}$ es impulsado en El Salvador desde el año 2010. Este se encuentra definido en el Plan Estratégico 20092014, dictado por el Ministerio de Economía (MINEC)*, a través de la Comisión Nacional de la Micro y Pequeña Empresa (CONAMY$\mathrm{PE})^{*}$. Esta estrategia surge como una respuesta novedosa de parte del Gobierno, a la necesidad de potenciar el crecimiento de la micro y pequeña empresa; así como al propósito de ampliar la cobertura de sus servicios.

Dicha iniciativa se ha diseñado para brindar atención empresarial, basada en el ciclo de desarrollo de una compañía (CONAMYPE, 2015). El modelo implica identificar en qué nivel del proceso se encuentra cada empresa, para así brindarle los apoyos y servicios que le permitirán seguir avanzando en su crecimiento. En respuesta a esto, las tecnologías de la información y la comunicación (TIC) 4 incorporadas por las Micro y Pequeñas Empresas (MYPE)*, deben estar relacionadas con el resto de servicios que las mismas ofrecen.

Entre las principales barreras para el acceso y uso de las TIC por parte de la MYPE, se encuentran: la falta de recursos económicos para invertir en equipo y en personal calificado; el desconocimiento del potencial que se tiene so- bre estas tecnologías para la mejora y la competitividad de la empresa; la accesibilidad y la falta de habilidades digitales.

Para superar esas barreras, se ha desarrollado una nueva cultura para difundir la utilización de las TIC entre los empresarios, con la finalidad de integrarse a una economía digitalizada. De igual manera, es necesario contar con líneas blandas de financiamiento para que las empresas de menor tamaño puedan adquirir el equipamiento requerido para ello. De igual forma, se ha impulsado una campaña de alfabetización digital, con recursos humanos y económicos, para garantizar la cobertura nacional. También se han habilitado espacios para la promoción, difusión, aprendizaje y acceso a las TIC; así como la contratación de jóvenes (nativos digitales) en las empresas MYPE.

\section{Normativa}

Las MYPES, en la actualidad, necesitan incorporar tecnología a sus estrategias de negocio para poder ser más productivas, competitivas y aumentar su grado de eficiencia. Es por eso que el Ministerio de Economía, a través de la Dirección de Innovación y Desarrollo Tecnológico (DIT), en conjunto con CONAMYPE, tiene un programa que facilita adoptar y sistematizar la gestión de la innovación en las micro y pequeñas empresas, como estrategia para mejorar su competitividad en los mercados nacional e internacional.

3*. Dentro del documento, los autores se referirán a este término mediante su acrónimo.

4. Entiéndase como tecnologías de la información y la comunicación. 
Específicamente, la Ley de Fomento, Protección y Desarrollo para la Micro y Pequeña Empresa (2014) tiene como objetivo fomentar la creación, protección, desarrollo y fortalecimiento de las Micro y Pequeñas Empresas. También busca contribuir en el fortalecimiento de su competitividad, con el fin de mejorar su capacidad generadora de empleos, y de brindar valor agregado a la producción. Todo esto con la finalidad de integrarlas a la economía formal del país, mediante la creación de un entorno favorable y competitivo, que permita a este sector empresarial un buen funcionamiento.

En el entorno empresarial actual, las TIC permiten a las organizaciones tejer redes de relaciones complejas con sus colaboradores, proveedores y clientes, para ofrecer productos y servicios competitivos. Al mismo tiempo que se establecen nuevos canales de relación, venta, mantenimiento y soporte.

Las tecnologías de información y comunicación son piezas fundamentales en el mundo económico y empresarial actual. Su implantación en la empresa permite la modernización y agilización de los procesos; incrementa los niveles de productividad, de innovación $\mathrm{y}$, en definitiva, aumenta la competitividad de la empresa en un mercado cada vez más globalizado y más competitivo (CONAMYPE, 2016). Las TIC son valiosas para la comunicación y procesamiento de datos, siendo una gran contribución al momento de tomar decisiones y acciones. Las empresas realmente pueden mejorar su productividad y competir más equitativamente en el mercado, si la información está disponible para ellas; y si cuentan con las herramientas para acceder a ella. (Martínez 2014).

\section{Estrategias}

Según CONAMYPE. (s.f.), las TIC impactan positivamente en las empresas dado que:

- Permiten tener información rápida y eficaz para la toma de decisiones.

- Reducen los costos de las transacciones (con clientes y proveedores).

- Permiten automatizar los procesos internos para volverse más eficiente.

- Facilitan los procesos de aprendizaje en su equipo humano.

Entre los beneficios que pueden obtener las micro y pequeñas empresas con la adopción de TIC se encuentran:

- Mayor número de canales de distribución. Posibilidad de acceso a vías rápidas de comunicación relativamente económicas, para llegar a un número mayor de potenciales clientes, incrementando sus posibilidades de ventas.

- Mejor gestión empresarial. Por medio de aplicaciones informáticas y dispositivos se pueden controlar todas aquellas variables y tareas relacionadas con la gestión del negocio: Inventarios, balance de ingresos y egresos, etc.

- Reducción de la carga administrativa. Las herramientas tecnológicas pueden significar la 
realización automatizada de tareas administrativas, cumplimiento de obligaciones tributarias, trámites bancarios, etc. Esto representa la posibilidad de destinar mayor tiempo a las actividades productivas y de mejora del negocio.

\section{- Posibilidad de iniciar procesos de innova-} ción. No se puede hablar de innovación sin hablar de TIC. Ambas cuestiones están estrechamente ligadas, puesto que las tecnologías de la información y comunicación, son el soporte de los actuales procesos de innovación empresarial (Revista ECONOMYPE).

Con base en lo anterior, surgen las siguientes interrogantes: ¿Qué resultados están obteniendo las empresas en relación al aumento de las ventas y gestión interna con el uso de las TIC? ¿Las MYPES que han adoptado la tecnología, la están utilizando de acuerdo al plan establecido? ¿Qué limitaciones se han generado en las MYPES con el uso de la tecnología? ¿Qué barreras les impiden a las empresas adoptar la tecnología? ¿Qué medidas se podrían tomar para optimizar el uso de las TIC?

Por ello se buscó determinar el nivel de influencia que tienen las TIC dentro de las empresas, específicamente en su gestión interna. De forma específica, se identificaron las competencias requeridas para el manejo de las tecnologías adoptadas por las empresas para potenciar su desempeño, así como las utilizadas para apoyar las ventas. Otro propósito del estudio fue establecer las limitaciones que se generan en las empresas respecto a la adopción de las TIC, en aras de pro- poner de aplicación de algunas de ellas dentro de la gestión empresarial de las MYPE.

Las variables que se abordaron en la investigación fueron: disponibilidad de recursos para la inversión en tecnología, barreras culturales, temor al cambio, desconocimiento de los sistemas informáticos, personal capacitado, gestión administrativa, tecnología de la información y comunicación; e influencia de las redes sociales para negocio. Cabe resaltar que, en el mundo empresarial, cada vez es más la demanda de profesionales eficientes, en todos los niveles y áreas de la empresa, para cumplir con la producción, el mercadeo y las finanzas.

La fundamentación teórica giró en torno al desarrollo de las capacidades productivas y competitivas de las medianas, pequeñas empresas; así como su articulación en las dinámicas de la economía territorial, nacional e internacional; especialmente en la aplicación de tecnologías.

\section{Metodología}

La investigación fue de tipo descriptiva. La población estuvo constituida por 228 empresas del departamento de Santa Ana, asesoradas por CDMYPE durante el año 2019. Los datos fueron proporcionados por el director de la sede de esta entidad, ubicada en la Universidad Católica de El Salvador (UNICAES). De la población, se seleccionó una muestra aleatoria simple del $30 \%$, equivalente a 68 instituciones. Se resalta que no se utilizaron técnicas estadísticas para el cálculo de la muestra. 
Las técnicas que se utilizaron fueron la encuesta y la entrevista, empleando como instrumento un cuestionario de 21 preguntas relacionadas al: mercado atendido, formación en TIC, nivel de estudios de los encargados, equipo informático utilizado, tecnología en internet, áreas de la empresa, medios para promocionar ventas, uso de redes sociales, aplicaciones usadas, y crecimiento empresarial por la implementación de tecnologías. El cuestionario fue suministrado a los encargados de las empresas.

\section{Resultados}

La información refleja que un $61.8 \%$ de las empresas están ubicadas el municipio de Santa Ana; un 3.8\% en el municipio de Metapán, un $2.9 \%$ en el municipio de Chalchuapa y un $1.5 \%$ el municipio de San Sebastián Salitrillo.

Todas ellas se dividen en los siguientes rubros:

Tabla 1. División de las empresas según su sector

\begin{tabular}{|c|c|}
\hline Rubros empresariales & $\begin{array}{c}\text { Porcentaje de } \\
\text { las empresas }\end{array}$ \\
\hline Industria y Comercio & $78 \%$ \\
\hline Solo Comercio & $18 \%$ \\
\hline Servicios & $4 \%$ \\
\hline
\end{tabular}

Otro aspecto relevante es que el $48.5 \%$ de las empresas investigadas tienen más de cinco años de estar operando; el 27.9\% tiene entre uno y dos años de existencia; mientras que el $13.2 \%$ aseguró estar laborando en el rango de dos a cuatro años y solo el $10.3 \%$ tiene menos de un año de funcionar.

La mayoría (94\%) de las empresas tienen entre 1 y 5 personas como empleados; y solo un $6 \%$ posee en sus planillas a más de 10 empleados. Todas las empresas atienden al mercado nacional; sin embargo, un $62 \%$ ya está exportando sus productos de manera ocasional no sistemática.

\section{Formación tecnológica}

Respecto a este aspecto, el 41\% de las empresas fomenta cada tres meses la adquisición de conocimientos relacionados a las tecnologías de la información y comunicación. En tanto, el $19 \%$ de las empresas capacita cada año a sus empleados. Sin embargo, un $28 \%$ de las instituciones nunca capacita a su personal. Es de resaltar que, en su mayoría, las capacitaciones recibidas son ofrecidas por CDMYPE.

Al consultárseles sobre el nivel de estudios que poseen las personas delegadas del manejo de las tecnologías, el 53\% manifestó que estos son profesionales universitarios; es decir, que de alguna manera dominan la temática. Por su parte, el $26 \%$ de los responsables tienen estudios a nivel de técnico; mientras que, el 18\% de las empresas manifestaron que dichos encargados no poseen certificación de estudios. Esta última situación puede ser contraproducente para los resultados positivos del negocio, ya que son ellos quienes manejan las TIC de la empresa.

En cuanto al equipo informático que se emplea, prácticamente casi todas las empresas (99\%), poseen un smartphone como equipo de trabajo. Entre los usos que le atribuyen al dispositivo se encuentran el tener conectividad empresarial y la facilidad de tener comunicación en tiempo real; además del manejo de los 
datos institucionales. Asimismo, utilizan otros tipos de dispositivos:

Tabla 2. Equipo informático utilizado por las empresas

\begin{tabular}{|c|c|}
\hline Tipo de equipo & Porcentaje \\
\hline Tablet & $29 \%$ \\
\hline Computadora de escritorio & $35 \%$ \\
\hline Laptop & $69 \%$ \\
\hline Smartphone & $99 \%$ \\
\hline
\end{tabular}

El total de las empresas, solo el $87 \%$ de ellas emplean la tecnología para hacer promoción, por medio de las redes sociales. Un $66 \%$ utiliza el correo electrónico; mientras que el 56\% promueve las ventas en línea, haciendo uso de páginas web (24\%). Este mismo porcentaje utiliza ese recurso para hacer compras en línea y el 13\% la utiliza para realizar pagos en línea. Solo el 2\% no utiliza internet.

\section{Utilidad}

Algunos de los usos que tiene la tecnología dentro de las empresas asesoradas por CDMYPE son: la facturación, contabilidad, inventarios, registros de ventas $\mathrm{y}$, principalmente dentro del mercadeo, en el caso de la promoción mediante redes sociales. En este último aspecto, resalta Facebook, como la red más empleada para estos fines, seguida de WhatsApp y finalmente, Instagram. La mayoría de los investigados manifestaron que no han recibido capacitación en el manejo de redes sociales; por su parte, otros aseguraron sí haberla recibido, especialmente conocimiento relacionado a las redes sociales antes mencionadas.
Herramientas tradicionales como página web y blogs, no son utilizadas por los micro-empresarios para publicar información de sus productos y/o servicios. Estos insumos son de uso frecuente en empresas medianas y grandes, que poseen recursos económicos, personal técnico y profesional que se encarga de su diseño, elaboración y mantenimiento.

Tabla 3. Áreas de utilización de las TIC

\begin{tabular}{|c|c|}
\hline Área de la empresa & Porcentaje \\
\hline Marketing & $88 \%$ \\
\hline Ventas & $81 \%$ \\
\hline Contabilidad & $41 \%$ \\
\hline Inventario & $41 \%$ \\
\hline Producción & $25 \%$ \\
\hline Facturación & $18 \%$ \\
\hline Otra & $5 \%$ \\
\hline
\end{tabular}

\section{Nivel de crecimiento}

Aunque las empresas utilicen las redes sociales más populares para promocionar sus productos y/o servicios, y hayan recibido capacitación en el manejo de las mismas, los resultados experimentados por los empresarios no están acorde a sus expectativas. Esto debido a que la promoción en estas herramientas no solo implica saber manejarlas, sino también conocimiento y experiencia en estrategias de promoción, a través de estos medios (ver tabla 4).

De igual forma, existen limitantes en el uso de las TIC. En este aspecto, las respuestas por parte de los empresarios son muy variadas, entre ellas, la falta de presupuesto o la no existencia de asesoría respecto al tema. Cabe señalar que el $18 \%$ de los empresarios manifestaron tener 
desconfianza de la tecnología; en tanto que otros piensan que se requiere de personal calificado para el empleo de ella (ver tabla 5).

Por otra parte, un $18 \%$ aseguró no solo no tener limitaciones, sino además poseer un alto grado de conocimientos sobre tecnología; seguido de un $15 \%$ que manifestó tener mucho conocimiento y un $35 \%$ de los empresarios que cataloga sus saberes en un punto intermedio. Sin embargo, al cuestionarles sobre si poseen un plan para el empleo de las tecnologías, el 26\% de ellos respondió que no lo tiene; es decir, en cuanto a este tema ellos improvisan. Esto en comparación a un 16\% que respondió positivamente al señalar que sus planes son altos en cuanto a la tecnología.
Cuando se les consultó sobre las razones que han provocado el incumplimiento de los planes, los empresarios manifestaron que esto se debe a la poca exploración que hacen de las redes, la falta de capacitación; un mal servicio en cuanto a la señal de conexión, el bajo presupuesto, la escasez de tiempo, tener equipo inadecuado y por la falta de interés.

\section{Aplicaciones utilizadas en comunicación y envío de documentos}

En este aspecto, WhatsApp es el medio de comunicación por excelencia (74\%), seguido de Messenger (53\%) y el correo electrónico (43\%); mientras que un 16\% no utiliza ninguna de estas. Sin embargo, se denota que no están al

Tabla 4. Crecimiento empresarial según implementación de TIC

\begin{tabular}{|c|c|c|c|c|}
\cline { 2 - 5 } \multicolumn{1}{c|}{} & \multicolumn{4}{c|}{ Porcentaje de crecimiento } \\
\hline Áreas de aplicación & Ninguno & Muy poco & Poco & Mucho \\
\hline Marketing & $13 \%$ & $6 \%$ & $12 \%$ & $26 \%$ \\
\hline Ventas & $4 \%$ & $4 \%$ & $9 \%$ & $12 \%$ \\
\hline Inventario & $53 \%$ & $3 \%$ & $6 \%$ & $9 \%$ \\
\hline Producción & $54 \%$ & $4 \%$ & $6 \%$ & $9 \%$ \\
\hline Contabilidad & $50 \%$ & $4 \%$ & $7 \%$ & $6 \%$ \\
\hline Facturación & $62 \%$ & $3 \%$ & $6 \%$ & $6 \%$ \\
\hline
\end{tabular}

Tabla 5. Limitantes en la utilización de TIC

\begin{tabular}{|l|c|}
\hline \multicolumn{1}{|c|}{ Motivos } & Porcentaje \\
\hline Falta de presupuesto para invertir en tecnología & $26 \%$ \\
\hline Falta de asesoría o asistencia técnica para su uso & $26 \%$ \\
\hline Requiere personal capacitado & $21 \%$ \\
\hline Falta de confianza en la tecnología & $18 \%$ \\
\hline Horarios no adecuados para las capacitaciones de CDMYPE & $8 \%$ \\
\hline Falta de tiempo para las capacitaciones que ofrece CDMYPE & $2 \%$ \\
\hline No la necesitan & $2 \%$ \\
\hline
\end{tabular}


tanto de otras aplicaciones como Skype, Dropbox, Slack, telegram y Google Drive.

Tabla 6. Aplicaciones utilizadas

\begin{tabular}{|c|c|}
\hline Categoría & Porcentaje \\
\hline WhatsApp & $74 \%$ \\
\hline Messenger de Facebook & $53 \%$ \\
\hline Correo Electrónico & $43 \%$ \\
\hline WhatsApp Business & $18 \%$ \\
\hline No se utilizan & $16 \%$ \\
\hline Google Drive & $6 \%$ \\
\hline Microsoft Outlook & $2 \%$ \\
\hline Telegram & $2 \%$ \\
\hline Skype & $0 \%$ \\
\hline Drive de Microsoft & $0 \%$ \\
\hline Dropbox & $0 \%$ \\
\hline Zoom & $0 \%$ \\
\hline Slack & $0 \%$ \\
\hline
\end{tabular}

\section{Discusión}

En la investigación se descubrió que aún persisten algunas barreras para la adopción y utilización de las TIC, siendo algunas de estas la falta de recursos para invertir en ellas y el desconocimiento de su potencial para la mejora y la competitividad de las empresas. Este último aspecto es, principalmente, un uso estratégico de las herramientas tecnológicas para realizar promoción y comercialización.

Por su parte, las redes sociales son herramientas con un alto número de usuarios, que no requieren de grandes conocimientos para su uso; pero para ser utilizadas en la promoción estratégica y dentro del área de negocios, se requiere de mucho conocimiento, experiencia e inversión. Con este estudio se logró identificar que los empresarios utilizan las TIC para la promoción, pero no están teniendo los resultados esperados. Por lo tanto, es necesario sensibilizarlos en el uso estratégico de las redes sociales y en aquellas herramientas de publicidad, promoción y comercialización que trabajan en conjunto con estas redes, como en el caso de Spotify, una herramienta de comercio electrónico que se puede integrar con Facebook. Al mismo tiempo, se debe dar seguimiento a su utilización y a su adecuada aplicación.

Existen aplicaciones especializadas para realizar registros contables, facturación y financieros, las cuales algunos empresarios ya están utilizando. Otros emplean herramientas como Excel de forma básica para llevar dichos registros. Es necesario fomentar el uso de estos insumos entre los empresarios que todavía no las han adoptado. También se deben identificar otras aplicaciones que ofrezcan según, las necesidades de los empresarios, que no impliquen un alto costo de licencias (software libre), ya el elemento financiero es una de las principales limitantes.

Algunas de las herramientas de comunicación, enfocadas en la realización de videoconferencia, que podrían potenciar el crecimiento del negocio son Skype y Zoom. Estas no se están utilizando y podrían ser muy útiles en el manteniendo de relaciones interactivas con los clientes. Se sugiere a los empresarios la adopción de un modelo acorde a sus necesidades, y que coadyuve a la mejora de la gestión 
empresarial para el acceso, el uso, la capacitación y la aplicación adecuada de los recursos y herramientas tecnológicas.

De esta forma se podrán obtener:

- Mayor número de canales de distribución. A través de las redes sociales, por ser un excelente multiplicador informativo; divulgándose de manera inmediata las promociones, precios y lugares de venta de los productos ofertados.
- Mejor gestión empresarial. Empleándose las aplicaciones informáticas en los inventarios y contabilidad.

- Reducción de la carga administrativa. Manejando datos oportunamente y haciendo negocios en línea.

- Posibilidad de iniciar procesos de innovación, al estarse actualizando en tecnología.

\section{Referencias}

Castillo, J. (2015). Comercio Electrónico y aspectos prácticos de Implementación con Magento. España: Cimapress. Recuperado de https://www.todostuslibros.com/libros/comercio-electronico-y-aspectos-practicos-de-implementacion-con-magento_978-84-16133-55-0

CONAMYPE. (s.f.). Estrategia para la inclusión digital de la MYPE. San Salvador, El Salvador. CONAMYPE. (s.f.). Plan estratégico de CONAMYPE 2015-2019. San Salvador, El Salvador.

CONAMYPE. (2014a). Ley de Fomento, Protección y Desarrollo para la Micro y Pequeña Empresa. San Salvador, El Salvador.

CONAMYPE (2014b). Política Nacional para el Desarrollo de la Micro y Pequeña Empresa. San Salvador, El Salvador.

Herradón, A. (2009). Marketing electrónico para PYMES. Como vender, promocionar y pensionarse en Internet. México: Editorial Alfaomega.

Laudon. K. (2009). E-comerce, negocios, tecnología, sociedad. México: Person Educación.

Martínez, M. (2012). Las TIC: una herramienta básica para el desarrollo de la MYPE. Revista Institucional de Comisión Nacional de la Micro y Pequeña Empresa ECONOMYPE, (1),4-5. Recuperado de https://issuu.com/mpdg/docs/economype 01

Ministerio de Economía-Dirección General de Estadísticas y Censos (23 de septiembre de 2011). Encuesta de Tecnologías. Recuperado de: http://www.digestyc.gob.sv/index.php/temas/ee/encuestas-economicas/encuesta-de-tecnologias.pdf

Rodríguez, I. (2017). Marketing Digital y comercio electrónico. España: Ediciones Pirámide. 
Romero, M. (2018). Vender en las plataformas digitales. España: ESIC Editorial.

Rovira, S.; Santoleri, P. y Stumpo, G. (2013). Incorporación de TIC en el sector productivo: uso y desuso de las políticas públicas para favorecer su difusión. En S. Rovira y G. Stumpo, Entre mitos y realidad. TIC, políticas públicas y desarrollo productivo en América Latina (17-50). Santiago, Chile: Naciones Unidas.

RTI International (2015). Perfil Sectorial: Tecnologías de la Información y Comunicación. Proyecto de USAID de Educación Superior para el Crecimiento Económico. San Salvador, El Salvador.

Ruano, L. (2018). Emprendimiento en el Entorno Digital. El Lanzamiento de una Startup. México: Alfaomega.

Somalo, I. (2017). El Comercio Electrónico, una guía completa para gestionar la venta online. Madrid: Editorial ESIC.

Unión Internacional de Telecomunicaciones (2015). Informe sobre medición de la sociedad de la información. Ginebra, Suiza.

World Economic Forum (2015a). The Global Information Technology Report 2015. Geneva.

World Economic Forum (2016b). The Global Information Technology Report 2016. Geneva. 